\title{
CARACTERIZAÇÃO DAS CONDIÇÕES DE MICROSSÍTIO DE ÁREAS EM RESTAURAÇÃO COM DIFERENTES IDADES ${ }^{1}$
}

\author{
Maria Isabel Ferreira Bertacchi², Pedro Henrique Santin Brancalion³, GilvanoEbling Brondani ${ }^{4}$, João \\ Carlos Medeiros ${ }^{5}$ e Ricardo Ribeiro Rodrigues ${ }^{2}$
}

\begin{abstract}
RESUMO - Os microssítios de regeneração são caracterizados por diversas combinações de atributos que representam condições que influenciam a germinação de sementes e o estabelecimento de plântulas. O conhecimento desses atributos pode contribuir para a determinação de metodologias adequadas de manejo, visando ao restabelecimento dos processos ecológicos nas áreas em processo de restauração. Dessa forma, o objetivo deste trabalho foi caracterizar e diferenciar as condições físico-químicas de microssítios de regeneração de áreas em processo de restauração florestal, visando identificar possíveis limitações físicas e químicas ao estabelecimento de espécies arbóreas nativas no sub-bosque. O estudo foi desenvolvido em reflorestamentos de espécies nativas com diferentes idades (10, 22 e 55 anos). Foi realizada a avaliação do grau de compactação, porosidade, umidade, conteúdo de matéria orgânica e nutrientes e granulometria do solo, bem como a massa de matéria seca de serapilheira e a cobertura do dossel de cada área de estudo. Houve aumento da cobertura do dossel, da porosidade, da umidade, do conteúdo de argila, da matéria orgânica e de outros nutrientes, e uma diminuição da compactação do solo, com o aumento da idade da restauração. Assim, conclui-se que, com a evolução da restauração, as condições de microssítio de regeneração estão se assemelhando gradativamente às presentes nos ecossistemas de referência, sendo este um aspecto positivo para que o recrutamento de espécies nativas seja favorecido ao longo do tempo.
\end{abstract}

Palavras-chave: Sítio de estabelecimento, Restauração florestal e Estabelecimento de plântulas.

\section{CHARACTERIZATION OF THE MICRO-SITE CONDITIONS FROM RESTORED AREAS WITH DIFFERENT AGES}

\begin{abstract}
Regeneration microsites are characterized by diverse combinations of attributes which assure the best conditions for seed germination and seedling establishment. By understanding these attributes, we can contribute to determining better management methodologies for reestablishing ecological process in sites under restoration. Thus, we sought to characterize and differentiate the micro-site conditions of restoration plantings to indentify likely physical-chemical limitations for the establishment of native tree species in the forest understory. This study was carried out in reforestation plantings with different ages (10, 22 and 55 years). The physical-chemical characterization of the micro-site of regeneration of the study areas was carried out by evaluating the soil compression level, porosity, humidity, organic matter and nutrients content and granulometry, as well as litter dry mass and canopy cover. An increase on the canopy cover and soil porosity,
\end{abstract}

\footnotetext{
${ }^{1}$ Recebido em 14.04.2012 aceito para publicação em 04.06.2012.

${ }^{2}$ Departamento de Ciências Biológicas, Laboratório de Ecologia e Restauração Florestal, Escola Superior de Agricultura "Luiz de Queiroz", ESALQ/USP, Brasil.E-mail: <belbertacchi@gmail.com>e <rrresalq@usp.br>.

${ }^{3}$ Departamento de Ciências Florestais, Escola Superior de Agricultura "Luiz de Queiroz", ESALQ/USP, Brasil. E-mail:<pedrob@usp.br>, ${ }^{4}$ Departamento de Engenharia Florestal, Universidade Federal do Mato Grosso, UFMT, Brasil. E-mail: <gebrondani@ yahoo.com.br>.

${ }^{5}$ Embrapa Arroz e Feijão, CNPAF, Brasil. E-mail: <medeiros.jc@gmail.com>.
} 
humidity, clay and organic matter content were observed in the oldest restored areas, as well as a decrease in soil compression. Thus, these findings demonstrated that the evaluated microsite properties are in process of restoration. Therefore, microsite conditions for seedling establishment become even more similar to reference ecosystems as restoration planting evolve.

Keywords: Site of establishment; Forest Restoration; Seedlings Establishment.

\section{INTRODUÇÃO}

O conceito de microssítio favorável para o estabelecimento, também conhecido como "safe site" na literatura inglesa, foi descrito originalmente por Harper et al. (1961) como o ambiente ao redor de uma semente que possui condições ambientais e ecológicas favoráveis para sua germinação e posterior emergência da plântula. Os microssítios são caracterizados por diversas combinações de atributos que asseguram um microclima favorável, adequada estrutura e textura do solo (BRADSHAW; CHADWICK, 1980; FOWLER, 1986, 1988), umidade e disponibilidade de nutrientes (KELLMAN, 1979; UHL et al., 1981), características da serapilheira (SCARIOT, 2000; MOLOFSKY; AUGSPURGER, 1992), condições de luz (URIARTE et al., 2010) e ausência de competidores, predadores e patógenos (HARPER, 1977). Assim, a disponibilidade de microssítios favoráveis interfere diretamente no estabelecimento de plântulas, o qual compreende os processos de emergência, mortalidade e crescimento inicial da plântula (PARCIAK, 2002). O estabelecimento pode representar a principal barreira para que uma espécie vegetal ocorra em diferentes ambientes (FENNER; THOMPSON, 2005).

O sucesso do estabelecimento de plântulas é geralmente limitado por uma baixa abundância de sementes ou por um número limitado de microssítios favoráveis (TURNBULL et al., 2000; URIARTE et al., 2010; NORGHAUER; NEWBERY, 2010). Porém, em áreas onde a dispersão de sementes não é um fator limitante, o estabelecimento de plântulas depende da disponibilidade de microssítios favoráveis (GRUBB, 1977; SVENNING; WRIGHT, 2005; BARBERÁ et al., 2006; DOUST et al., 2006).

A importância de tais sítios e de seus atributos tem sido enfatizada (URBANSKA, 2000), mas eles raramente são avaliados em áreas em processo de restauração (ELMARSDOTTIR et al., 2003). Assim, o conhecimento desses atributos e seus efeitos sobre o estabelecimento de plântulas devem ser levados em consideração, pois podem contribuir para aumentar a efetividade dos projetos de restauração (ELMARSDOTTIR et al., 2003).

No caso de haver limitações de microssítio ao estabelecimento de plântulas, é fundamental identificar qual a contribuição de cada atributo do microssítio, para que, então, se possa manipulá-lo, criando condições mais adequadas ao estabelecimento (DOUST et al., 2006).

Assim, os objetivos deste trabalho foram: a) caracterizar e diferenciar as condições físico-químicas de microssítios de áreas em processo de restauração florestal; b) identificar as limitações ao estabelecimento de espécies arbóreas nativas no sub-bosque; e c) diagnosticar se, com o tempo, as áreas restauradas com espécies nativas adquirem condições de microssítio mais próximas daquelas ocorrentes em remanescentes naturais.

\section{MATERIAL E MÉTODOS}

\subsection{Locais de estudo}

O estudo foi desenvolvido em três áreas de florestas em processo de restauração com diferentes idades, localizadas no interior do Estado de São Paulo: Santa Bárbara d'Oeste $\left(22^{\circ} 43^{\prime} \mathrm{S} \quad 47^{\circ} 20^{\prime} \mathrm{W}\right.$; 10 anos de implantação da restauração), Iracemápolis $\left(22^{\circ} 35^{\prime} \mathrm{S}\right.$ $47^{\circ} 31^{\prime} \mathrm{W}$; 22 anos) e Cosmópolis ( $22^{\circ} 39^{\prime} \mathrm{S} \quad 47^{\circ} 12^{\prime} \mathrm{W}$; 55 anos). As três áreas são geograficamente próximas umas das outras e encontram-se praticamente isoladas na paisagem regional onde se inserem, tendo uma matriz predominantemente composta por monocultura de canade-açúcar. As restaurações com 10, 22 e 55 anos foram implantadas com alta diversidade de espécies (80, 140 e 71 espécies, respectivamente), em comparação com as cerca de 70 espécies normalmente encontradas em florestas ripárias (RODRIGUES et al., 2011), e atualmente possuem dossel com cerca de $20 \mathrm{~m}$ de altura.

As três áreas inserem-se na região de ocorrência natural da Floresta Estacional Semidecidual (VELOSO, 1992), uma das formações que compõem o bioma Mata 
Atlântica, com clima do tipo Cwa - tropical de altitude, com inverno seco e verão chuvoso, segundo a classificação de Köeppen (1948), e possuem relevo suave e ondulado.

\subsection{Caracterização do microssítio de estabelecimento nas áreas de estudo}

Em cada área de estudo foram delimitados 10 pontos de amostragem, dispostos longitudinalmente, um após o outro, a cada $20 \mathrm{~m}$. As amostras para a caracterização das áreas de estudo foram coletadas ao redor de cada ponto de amostragem.

\subsubsection{Compactação do solo}

O grau de compactação do solo foi quantificado por meio da medida de densidade do solo. Foram coletadas amostras de solo, em duas profundidades, $0-5 \mathrm{~cm} \mathrm{e}$ $15-20 \mathrm{~cm}$, ao redor de cada ponto de amostragem, nas três áreas de estudo, utilizando-se cilindros volumétricos de $100 \mathrm{~cm}^{3}$ ( $5 \mathrm{~cm}$ de diâmetro $x 5 \mathrm{~cm}$ de altura). O excesso de solo foi eliminado dos bordos com o auxílio de uma espátula, de forma que o volume do cilindro fosse exatamente igual ao volume de solo em seu interior. As amostras de solo foram acondicionadas em sacos plásticos e, posteriormente, colocadas em estufa a $105^{\circ} \mathrm{C}$, por $48 \mathrm{~h}$, para então se determinar sua densidade. A densidade do solo foi determinada conforme metodologia proposta por Blake e Hartge (1986).

\subsubsection{Porosidade do solo}

A porosidade do solo foi determinada utilizando-se as mesmas amostras usadas na avaliação de sua densidade. A macroporosidade do solo foi avaliada em mesa de areia ajustada a $60 \mathrm{~cm}$ de tensão de sucção (TOPP; ZEBCHUK, 1979). A porosidade total foi calculada pela razão densidade do solo/densidade de partículas, e a microporosidade foi obtida pela diferença entre porosidade total e macroporosidade (EMBRAPA, 1997).

\subsubsection{Umidade do solo (umidade gravimétrica)}

As amostras foram retiradas em duas profundidades, 0-20 cm e $20-40 \mathrm{~cm}$, ao redor de cada ponto de amostragem, nas três áreas estudadas. As coletas de amostras foram feitas por um período de quatro meses (março a junho de 2010), a cada 15 dias, totalizando sete coletas. As amostras foram secas em estufa a $110^{\circ} \mathrm{C}$ por $48 \mathrm{~h}$, e seus pesos de solo úmido e seco foram determinados. A umidade gravimétrica $(U G)$ foi obtida pela relação:

$$
U G=(m u-m s) / m s \times 100
$$

em que $U G$ é igual à umidade gravimétrica do solo; $m u$, massa do solo úmido; e $m s$, massa do solo seco.

\subsubsection{Massa seca de serapilheira}

Em cada área de estudo foram coletadas 10 amostras de serapilheira em parcelas de $0,5 \mathrm{~m}$ x $0,5 \mathrm{~m}$ localizadas a cerca de $1 \mathrm{~m}$ dos pontos de amostragem. O material que estava acima da porção mineral do solo foi coletado no campo e acondicionado em saco de papel. Posteriormente, foram secos em estufa de circulação de ar a $80^{\circ} \mathrm{C}$, por $48 \mathrm{~h}$, e pesados.

\subsubsection{Análise química do solo}

Em cada área de estudo, 10 amostras de solo (0-20 cm de profundidade) foram coletadas, a cerca de $1 \mathrm{~m}$ dos pontos de amostragem. As amostras foram individualmente submetidas à análise no Laboratório de Análises Químicas (ESALQ/USP). As concentrações de matéria orgânica (MO), K (mmol/ $/ \mathrm{kg}), \mathrm{P}(\mathrm{mg} / \mathrm{kg}), \mathrm{Ca}$ ( $\mathrm{mmol} / \mathrm{kg}), \mathrm{Mg}$ ( $\mathrm{mmol} / \mathrm{kg}$ ), $\mathrm{Al}$ (mmol/ $/ \mathrm{kg}), \mathrm{HzAl}$ ( $\mathrm{mmol} / \mathrm{kg}$ ) e valores de $\mathrm{pH}$ de cada área de estudo foram determinadas.

\subsubsection{Granulometria do solo}

Em cada área de estudo foram coletadas 10 amostras de solo (0-20 $\mathrm{cm}$ de profundidade), a cerca de $1 \mathrm{~m}$ dos pontos de amostragem. As amostras foram submetidas à análise granulométrica (areias $(\mathrm{g} / \mathrm{kg})$, silte $(\mathrm{g} / \mathrm{kg})$ e argilas (g/kg)), no Laboratório de Física do Solo (ESALQ/USP).

\subsubsection{Cobertura do dossel}

Um densiômetro esférico côncavo (LEMMON, 1957) foi utilizado para determinar o nível de cobertura do dossel em cada um dos 10 pontos de amostragem, nas três áreas de estudo. As medições foram realizadas no pico da estação seca, durante o mês de agosto. Foram tomadas, por ponto, quatro medidas, em quatro orientações geográficas distintas (norte, sul, leste e oeste), a cerca de 1,3 m do solo, sempre por uma mesma pessoa. Cada quadrante do densiômetro foi dividido em quatro e sistematicamente contados quantos quartos do quadrante refletiam o dossel. O total dos quadrantes foi somado e multiplicado por 1,04, obtendo-se dados de porcentagem de abertura/cobertura do ponto. A porcentagem de cobertura do dossel em cada bloco foi, então, obtida por meio da média da cobertura amostrada em cada um dos pontos cardeais (LEMMON, 1957; SUGANUMA et al., 2008).

Revista Árvore, Viçosa-MG, v.36, n.5, p.895-905, 2012 


\subsubsection{Densidade e riqueza de regenerantes}

Em cada área de estudo foram marcadas 40 parcelas de $1 \mathrm{mx} 1 \mathrm{~m}$. Foram tomadas quatro medidas por ponto, em quatro coordenadas geográficas distintas (norte, sul, leste e oeste), cada uma distante $4 \mathrm{~m}$ de cada ponto de amostragem. Dentro de cada parcela, foram amostrados todos os indivíduos regenerantes com até $50 \mathrm{~cm}$ de altura, os quais foram separados em morfoespécies. Os indivíduos de espécies exóticas, que estavam em desequilíbrio na área, foram excluídos das análises, evitando assim, uma interpretação equivocada dos resultados.

\subsection{Análise dos dados}

A coleta dos dados de porcentagem de cobertura do dossel, a massa de matéria seca de serapilheira e a análise química e granulométrica do solo seguiram o modelo de delineamento em blocos ao acaso. Quanto aos dados de densidade, porosidade e umidade, o experimento seguiu o modelo de blocos ao acaso no arranjo bifatorial (3x2). A detecção da diferenciação entre as áreas de estudo em relação às características do microssítio deu-se por análise de variância (ANOVA, $P<0,01$ e $P<0,05)$ e, quando encontradas diferenças significativas, as médias foram comparadas pelo teste de Tukey $(P<0,05)$. As análises foram realizadas pelo software estatístico SAS (SAS INSTITUTE, 2002).

\section{RESULTADOS}

\subsection{Cobertura do dossel (\% CD)}

A área restaurada há 10 anos (Santa Bárbara d'Oeste) apresentou porcentagem de cobertura do dossel significativamente menor do que as áreas restauradas há 22 e 55 anos, consideradas estatisticamente semelhantes (Figura 1).

\subsection{Densidade e riqueza de regenerantes}

A avaliação da densidade de indivíduos regenerantes nas três áreas de estudo evidenciou maior densidade e diversidade de regenerantes na restauração mais antiga (Figura 1).

\subsection{Porosidade e densidade do solo}

As áreas em restauração há 10 e 22 anos apresentaram maiores valores de macroporosidade na camada mais superficial do solo $(0-5 \mathrm{~cm})$, em relação à camada mais profunda $(15-20 \mathrm{~cm})$ (Tabela 1$)$. Quanto
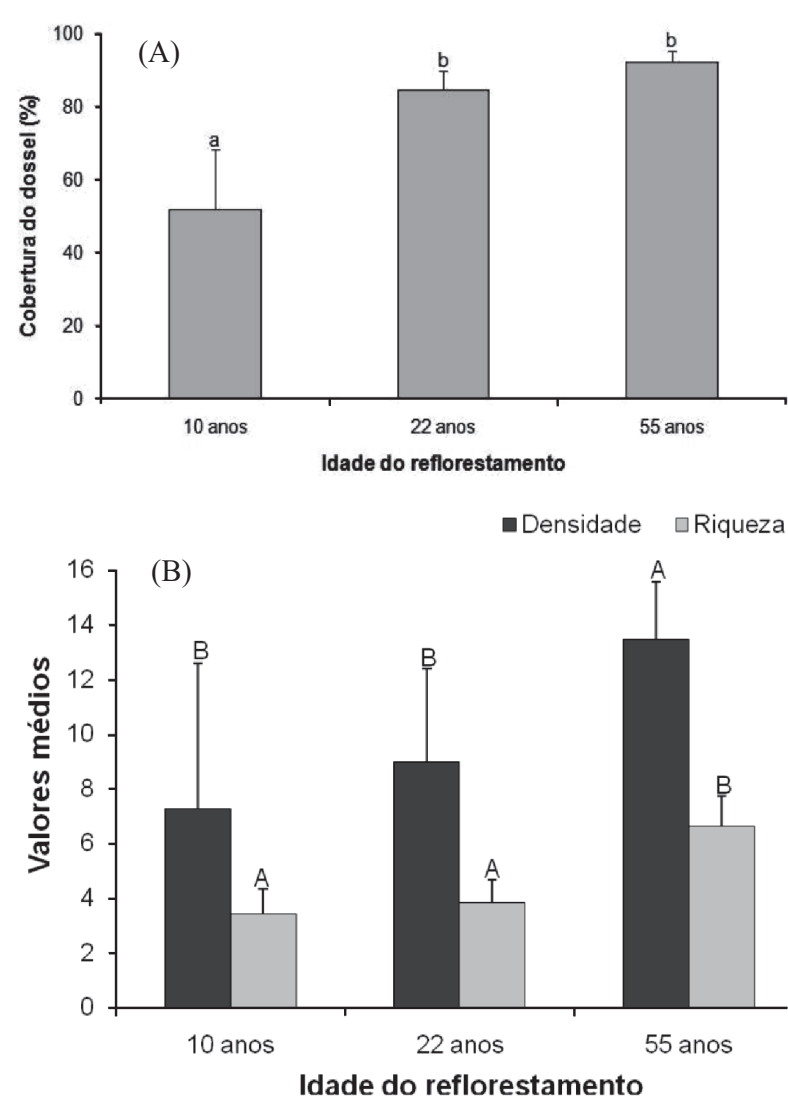

Figura 1 - Restaurações com 10, 22 e 55 anos de idade. A) Porcentagem de cobertura do dossel. B) Valores médios de densidade e riqueza de regenerantes. Cada barra e linha vertical representam, respectivamente, a média \pm o desvio-padrão. As médias seguidas por letras iguais não diferem significativamente entre si, pelo teste de Tukey a $5 \%$ de probabilidade de erro.

Figure 1 - Restorations at 10, 22 and 55 years of implementation. A) Percent canopy cover. B) Average values of density and richness of regenerants. Each bar and vertical lines represent the average \pm standard deviation, respectively. Means followed by same letters do not differ significantly by Tukey test at $5 \%$ probability.

aos microporos, verificaram-se menores valores, em ambas as profundidades, entre a área mais jovem (10 anos) e as demais restaurações (Tabela 1). As amostras coletadas superficialmente $(0-5 \mathrm{~cm})$ apresentaram maior porosidade total, em relação às amostras mais profundas (15-20 cm) (Tabela 2). Inversamente, a densidade do solo foi significativamente maior na restauração com 10 anos 
Tabela 1 - Valores médios da macroporosidade, microporosidade, porosidade total e densidade do solo. Table 1 -Average values for macroporosity, microporosity, total porosity and soil density.

\begin{tabular}{lcccccc}
\hline $\begin{array}{l}\text { Idade da } \\
\text { área }\end{array}$ & \multicolumn{2}{c}{ Macroporosidade } & \multicolumn{2}{c}{ Microporosidade } & Porosidade total & Densidade \\
\hline & $0-5$ & $15-20$ & $0-5$ & $15-20$ & & \\
10 anos & $0,120 \pm 0,035 \mathrm{Aa}$ & $0,058 \pm 0,027 \mathrm{Ab}$ & $0,353 \pm 0,017 \mathrm{Ba}$ & $0,354 \pm 0,014 \mathrm{Ba}$ & $0,442 \pm 0,041 \mathrm{~B}$ & $1,480 \pm 0,109 \mathrm{~A}$ \\
22 anos & $0,124 \pm 0,033 \mathrm{Aa}$ & $0,068 \pm 0,026 \mathrm{Ab}$ & $0,433 \pm 0,017 \mathrm{Aa}$ & $0,443 \pm 0,025 \mathrm{Aa}$ & $0,534 \pm 0,040 \mathrm{~A}$ & $1,233 \pm 0,105 \mathrm{~B}$ \\
55 anos & $0,113 \pm 0,038 \mathrm{Aa}$ & $0,103 \pm 0,046 \mathrm{Aa}$ & $0,450 \pm 0,042 \mathrm{Aa}$ & $0,420 \pm 0,011 \mathrm{Aa}$ & $0,540 \pm 0,041 \mathrm{~A}$ & $1,216 \pm 0,109 \mathrm{~B}$ \\
\hline
\end{tabular}

Nas colunas, médias seguidas por mesma letra maiúscula e, nas linhas, médias seguidas por mesma letra minúscula não diferem significativamente pelo teste de Tukey a 5\% de probabilidade de erro. Dados apresentados como média \pm desvio-padrão.

(Tabela 1), e maiores valores de densidade foram encontrados nas amostras mais profundas de solo $(15-20 \mathrm{~cm})$ (Tabela 2).

\subsection{Granulometria do solo}

A quantidade de areia, silte e argila foi significativamente diferente $(\mathrm{p}<0,01)$ entre as três restaurações estudadas. A quantidade de areia e o teor de silte foram, respectivamente, inversa e diretamente proporcionais à idade do reflorestamento. A quantidade de argila foi maior no plantio de 22 anos. O reflorestamento de 55 anos apresentou quantidade próxima, porém significativamente menor. Os menores teores de argila foram encontrados na floresta restaurada mais jovem (Figura 2).

\subsection{Umidade do solo}

O padrão de variação do teor de água no solo foi o mesmo nas três áreas de estudo. Os valores médios de umidade foram significativamente diferentes em relação

Tabela 2 - Valores médios da porosidade total e densidade do solo, em relação à profundidade da amostra coletada, considerando-se os dados das três áreas avaliadas e restaurações com 10, 22 e 55 anos.

Table 2 -Average values of total porosity and soil density related to depth of collected sample. All three areas (10, 22 and 55 year restoration) were considered in this analysis.

\begin{tabular}{ccc}
\hline Profundidade $(\mathrm{cm})$ & Porosidade total & Densidade \\
\hline $0-5$ & $0,530 \pm 0,049 \mathrm{~A}$ & $1,245 \pm 0,131 \mathrm{~B}$ \\
$15-20$ & $0,481 \pm 0,062 \mathrm{~B}$ & $1,374 \pm 0,164 \mathrm{~A}$ \\
\hline
\end{tabular}

Nas colunas, médias seguidas por mesma letra não diferem significativamente pelo teste de Tukey a $5 \%$ de probabilidade de erro. Dados apresentados como média \pm desvio-padrão
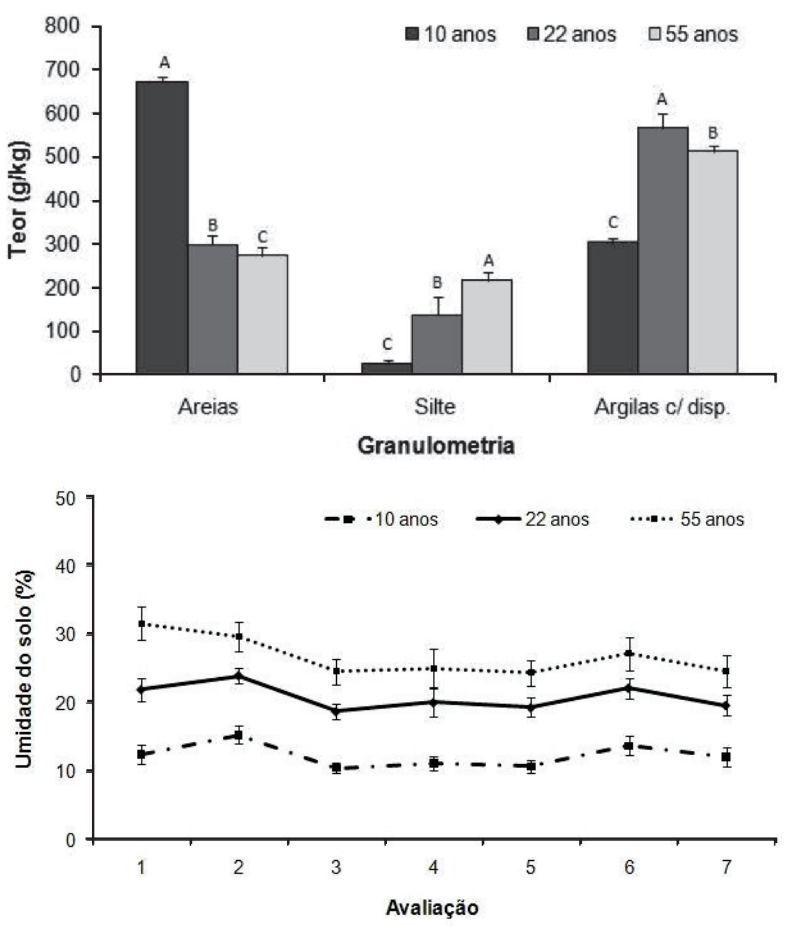

Figura 2 - Restaurações com 10, 22 e 55 anos de idade. A) Distribuição de tamanho de partícula (areia, silte e argila). B) Valores de umidade gravimétrica ao longo de sete avaliações, realizadas nos meses de março a junho de 2010. Cada barra e linha vertical representam, respectivamente, a média \pm o desvio-padrão. As médias seguidas por letras iguais não diferem significativamente entre si, pelo teste de Tukey a $5 \%$ de probabilidade de erro.

Figure 2-Restorations at 10, 22 and 55 years of implementation. A) Distribution of the particle (sand, silt, clay) size. B) Gravimetric humidity values over the seven evaluations carried out from March to June 2010. Each bar and vertical lines represent the average \pm standard deviation, respectively. Means followed by same letters do not differ significantly by Tukey test at $5 \%$ probability.

Revista Árvore, Viçosa-MG, v.36, n.5, p.895-905, 2012 
à idade da área restaurada e, de maneira geral, o solo da restauração mais antiga mostrou maior capacidade de armazenamento de água (Figura 2). Diferentemente, a restauração mais jovem apresentou menor quantidade de água armazenada ao longo de todo o período de avaliação.

Embora a avaliação da umidade gravimétrica do solo tenha sido conduzida por um período restrito de quatro meses, espera-se que esse padrão se mantenha o mesmo em outras épocas do ano.

\subsection{Análise química do solo}

Os maiores valores de pH, P, Ca, Mg e matéria orgânica (MO) foram encontrados na área restaurada mais antiga, de 55 anos. A área de 10 anos apresentou maior valor de $\mathrm{K}$ no solo, enquanto a restauração de idade intermediária (22 anos), maior valor de $\mathrm{Al}$ e $\mathrm{H}^{+}$ Al no solo (Tabela 3 ).

\subsection{Massa seca de serapilheira (MSS)}

Não foram observadas diferenças significativas entre as áreas e entre blocos, em relação ao parâmetro avaliado, a $5 \%$ de probabilidade de erro, pela ANOVA.

\section{DISCUSSÃO}

A avaliação da cobertura do dossel e da densidade e riqueza de regenerantes das três restaurações estudadas evidenciou uma evolução estrutural das áreas conforme a idade, resultado esperado para restaurações fundamentadas na sucessão secundária (RODRIGUES; GANDOLFI, 2004). As restaurações mais antigas apresentaram valores de cobertura próximos ao encontrado por Meira-Neto et al. (2005), em um remanescente de Floresta Estacional Semidecidual localizado em Viçosa, MG, na mesma época do ano (70\%).
A cobertura promovida pelo dossel influencia a quantidade, qualidade e distribuição temporal e espacial da luz, determinando níveis diferenciados de umidade do ar, temperatura e condições de umidade do solo (JENNINGS et al., 1999), além de promover a interceptação das chuvas, reduzindo o impacto direto sobre o solo. Assim, a cobertura florestal de uma área apresentase fortemente relacionada com a manutenção das propriedades físicas e químicas do solo (AN et al., 2008; GONÇALVES et al., 2003). Dessa forma, o solo de restaurações mais recentes, além de mais suscetível à erosão, apresenta tempo menor de recuperação de seus atributos, quando comparado com restaurações mais antigas, fazendo que geralmente apresente maior nível de degradação. No entanto, há que se considerar que as avaliações foram realizadas em um período restrito de quatro meses do ano, na transição da estação chuvosa para a estação seca. Como se espera que nesse período ocorram maiores mudanças nos padrões de estabelecimento de plântulas, acredita-se que esses padrões possam ser diretamente afetados pelo nível de cobertura do dossel.

A densidade e porosidade do solo são propriedades relacionadas com alterações no volume de solo e, portanto, altamente relacionadas com a sua compactação. A maior densidade encontrada na restauração mais recente confirma os resultados encontrados por Hajabbasi et al. (1997), em que áreas que apresentaram maior nível de degradação da cobertura florestal exibem maior densidade do solo. Sahani e Behera (2001) concluíram que o desmatamento acarreta perda estrutural do solo (aumento da densidade e diminuição da porosidade), o que indica aumento da compactação.

Nas áreas estudadas, observou-se uma diminuição gradual da densidade conforme a evolução da área. Essa diferença chegou a quase $20 \%$ entre as restaurações

Tabela 3 - Valores médios de $\mathrm{pH}\left(\mathrm{CaCl}_{2}\right)$, potássio $(\mathrm{K})$, fósforo $(\mathrm{P})$, cálcio $(\mathrm{Ca})$, magnésio $(\mathrm{Mg})$, alumínio $(\mathrm{Al})$, hidrogênio mais alumínio $\left(\mathrm{H}^{+} \mathrm{Al}\right)$ e matéria orgânica $(\mathrm{MO})$ no solo das áreas de estudo (restaurações com 10, 22 e 55 anos).

Table 3 - Average values of $\mathrm{pH}\left(\mathrm{CaCl}_{2}\right)$, potassium $(\mathrm{K})$, phosphorus $(\mathrm{P})$, calcium $(\mathrm{Ca})$, magnesium $(\mathrm{Mg})$, aluminum $(\mathrm{Al})$, hydrogenplus aluminum $\left(H^{+} A l\right)$ and organic matter in the soil from the studied areas $(10,22$ and 55 years of restoration).

\begin{tabular}{|c|c|c|c|c|c|c|c|c|}
\hline $\begin{array}{c}\text { Idade da } \\
\text { área (anos) }\end{array}$ & $\begin{array}{c}\mathrm{pH} \\
\left(\mathrm{CaCl}_{2}\right)\end{array}$ & $\begin{array}{c}\mathrm{K} \\
(\mathrm{mmol} / \mathrm{Kg}) \\
\end{array}$ & $\begin{array}{c}\mathrm{P} \\
(\mathrm{mg} / \mathrm{Kg})\end{array}$ & $\begin{array}{c}\mathrm{Ca} \\
(\mathrm{mmol} / \mathrm{Kg})\end{array}$ & $\begin{array}{c}\mathrm{Mg} \\
(\mathrm{mmol} / \mathrm{Kg})\end{array}$ & $\begin{array}{c}\mathrm{Al} \\
(\mathrm{mmol} / \mathrm{Kg})\end{array}$ & $\begin{array}{c}\mathrm{H}^{+} \mathrm{Al} \\
(\mathrm{mmol} / \mathrm{Kg})\end{array}$ & MO \\
\hline 10 & $4,52 \mathrm{~B}$ & $5,21 \mathrm{~A}$ & $3,92 \mathrm{~B}$ & $9,00 \mathrm{~B}$ & $5,40 \mathrm{~B}$ & $5,87 \mathrm{~B}$ & $0,046 \mathrm{C}$ & $17,30 \mathrm{C}$ \\
\hline 22 & $4,15 \mathrm{~B}$ & $0,75 \mathrm{C}$ & $16,73 \mathrm{~A}$ & $10,70 \mathrm{~B}$ & $4,50 \mathrm{~B}$ & $14,29 \mathrm{~A}$ & $0,074 \mathrm{~A}$ & $26,21 \mathrm{~B}$ \\
\hline 55 & $5,23 \mathrm{~A}$ & $1,60 \mathrm{~B}$ & $24,46 \mathrm{~A}$ & $58,90 \mathrm{~A}$ & $19,10 \mathrm{~A}$ & $4,61 \mathrm{~B}$ & $0,057 \mathrm{~B}$ & $39,19 \mathrm{~A}$ \\
\hline
\end{tabular}

Nas colunas, médias seguidas por mesma letra não diferem significativamente pelo teste de Tukey a $5 \%$ de probabilidade de erro.

Revista Árvore, Viçosa-MG, v.36, n.5, p.895-905, 2012 
de 10 e 55 anos e pode ser atribuída ao tempo de restauração das áreas. Além disso, indica uma recuperação da estrutura do solo, conforme a evolução do processo de restauração. A abundância e distribuição das raízes nas camadas de solo influem acentuadamente na estruturação do solo (GARCIA et al., 1994; GONÇALVES et al., 2003; PÉREZ-BEJARANO et al., 2010), podendo esse ser um dos mecanismos que resultaram na melhoria da estrutura do solo ao longo do processo de restauração.

Os valores de densidade encontrados nas restaurações mais antigas ( 22 e 55 anos) estão abaixo do valor considerado restritivo $\left(1,40 \mathrm{Mg} \cdot \mathrm{m}^{-3}\right)$ ao crescimento radicular em solos das classes texturais deste estudo (ARSHAD et al., 1996). Mesmo a camada subsuperficial do solo $(15-20 \mathrm{~cm})$ das três áreas, que apresentou maior grau de compactação, ainda apresenta valores abaixo do considerado restritivo ao crescimento radicular. Em contrapartida, o valor encontrado na restauração com 10 anos $\left(1,48 \mathrm{Mg} \cdot \mathrm{m}^{-3}\right)$ ficou acima do limite crítico, indicando uma possível limitação ao estabelecimento de plântulas (Tabela 1).

A menor densidade do solo nas restaurações mais antigas ( 22 e 55 anos) pode estar diretamente associada à estruturação provocada no solo pelo acúmulo de carbono orgânico (CARPENEDO; MIELNICZUK, 1990; ARVIDSSON, 1998) e pela capacidade do solo de se regenerar com o passar do tempo (SEYBOLD et al., 1999). De maneira inversa à densidade, a porosidade do solo é drasticamente reduzida com a compactação (DIAS JR.; PIERCE, 1996). Assim, a maior densidade e menor porosidade do solo, como encontrado na restauração mais recente, podem ser consequências da perda de cobertura florestal (SAHANI e BEHERA, 2001). Além disso, os menores valores de porosidade total e a compactação do solo da restauração com 10 anos podem estar relacionados ao histórico de uso da área antes da implantação do reflorestamento (cultivo de cana-de-açúcar) e ao tempo limitado que essa área teve para se recuperar, em comparação com os reflorestamentos mais antigos (22 e 55 anos).

Os valores de macroporosidade da camada superficial do solo, em todas as áreas, ficaram em torno de $0,12 \mathrm{~m}^{3} \mathrm{~m}^{-3}$ (Tabela 1), sendo esse valor maior que o mínimo necessário para um adequado desenvolvimento dos vegetais, que é de $0,10 \mathrm{~m}^{3} \mathrm{~m}^{-3}$ (BAVER, 1949; VOMOCIL; FLOCKER, 1961; GRABLE; SIEMER, 1968; KIEHL, 1979; GUPTA;ALLMARAS, 1987; REICHERT et al., 2003). Porém, em maiores profundidades (15$20 \mathrm{~cm}$ ), as duas restaurações mais recentes (10 e 22 anos) apresentaram valores menores que o valor mínimo, apontando uma possível limitação ao desenvolvimento das plantas (REICHERT et al., 2003).

Apesar de os valores encontrados nas camadas superficiais indicarem a recuperação desse atributo, os valores de macroporosidade na camada subsuperficial das restaurações mais recentes reforçam a degradação do solo nessas áreas. Esses dados corroboram os resultados obtidos por Sahani e Behera (2001), em que em áreas degradadas foram encontrados menores valores de macroporosidade. Além disso, um menor valor de microporosidade na área mais jovem aponta para uma menor capacidade de retenção de água no solo.

Os resultados das análises granulométricas das áreas de estudo estão relacionados com o tipo de solo, ao manejo aplicado a esse solo antes da implantação da restauração e a recuperação do solo, conforme a evolução do reflorestamento. As características da textura do solo são de suma importância para a recuperação das áreas restauradas, pois essas características se apresentam correlacionadas com a distribuição de muitas das espécies em florestas (RODRIGUES et al., 2007).

Sahani e Behera (2001) relataram diferença entre a textura do solo de florestas naturais e áreas desmatadas e atribuíram essa diferença à degradação sofrida pelo solo. Nas áreas desmatadas, foram verificados maior conteúdo de areia e silte e menor de argila. $\mathrm{O}$ aumento da erosão leva a uma perda ou remoção das partículas de argila (LAL, 1976, 1989; NARAIN et al., 1990). A perda de argila como impacto negativo do desmatamento foi reportada também em diversos estudos (BASU; BEHERA, 1993; PHASAD et al., 1994; ISLAM; WEIL, 2000; HAJABBASI et al., 1997; SAHANI; BEHERA, 2001), os quais corroboram as tendências observadas nesta pesquisa. O reflorestamento mais recente apresentou maior conteúdo de areia e menor de argila, enquanto que a área mais antiga exibiu conteúdos inversos de areia e argila. Porém, o conteúdo de silte mostrou-se inverso ao encontrado por Sahani e Behera (2001).

A idade da restauração foi fator fundamental para definir o volume de água armazenado pelo solo. Essa influência da idade da restauração na capacidade do solo em armazenar água está relacionada ao conteúdo de carbono orgânico do solo. Com o passar dos anos, o não revolvimento do solo e o acúmulo de resíduos

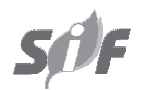

Revista Árvore, Viçosa-MG, v.36, n.5, p.895-905, 2012 
orgânicos na superfície provocam aumento na quantidade de matéria orgânica. Como a matéria orgânica tem capacidade de armazenar um volume elevado de água, solos com conteúdo de matéria orgânica mais elevado são capazes de armazenar mais água e, consequentemente, disponibilizar maior volume desta para as plantas. Adicionalmente, o aumento da cobertura de dossel com o incremento da idade do reflorestamento contribui para o armazenamento de água no solo, por diminuir a evaporação potencial.

Existem outros fatores que influenciam no armazenamento de água nos solos. Saxton e Rawls (2006) mostraram relação da umidade do solo com a sua textura, ou seja, em solos com maior conteúdo de argila, o volume de água armazenado é maior. No entanto, dos três solos estudados, aquele com maior conteúdo de argila foi o do reflorestamento de 22 anos, ficando à frente do reflorestamento de 55 anos. Dessa forma, o conteúdo de matéria orgânica desses solos parece ser o fator determinante para definir a capacidade de retenção de água nas áreas em processo de restauração estudadas.

Da mesma forma que as outras propriedades do solo avaliadas, a maioria dos nutrientes teve sua concentração gradativamente aumentada com a evolução da restauração. A intensa lixiviação sofrida pelo solo de áreas degradadas resulta em perda de nutrientes (HAJABBASI et al., 1997; ZHENG et al., 1997; SAHANI; BEHERA, 2001; LAL, 2002) e causa grandes implicações sobre a sua fertilidade (GONÇALVES et al., 2003; ZHENG, 2005; ZHANG et al., 2006).

Dessa forma, o pequeno aporte de substrato orgânico, devido à ausência de vegetação, contribuiu com esses baixos índices de matéria orgânica registrados em áreas degradadas (SAHANI; BEHERA, 2001). Esses dados corroboram com os resultados encontrados neste estudo, onde a restauração mais recente apresentou menor cobertura do dossel e, consequentemente, menor conteúdo de matéria orgânica no solo.

Diferentemente dos outros nutrientes encontrados, houve maior concentração de $\mathrm{K}^{+}$na restauração mais nova. Esse fato pode ser explicado pela frequente aplicação de fertilizantes na região, segundo constatação em campo, e deslocamento desses via enxurrada para a área em restauração, vizinha a cultivos de cana-deaçúcar que utilizam fertirrigação com vinhaça e adubação com torta de filtro, ambos muito ricos em potássio.
Além da perda de nutrientes por lixiviação, uma menor atividade e biomassa microbiana em áreas degradadas e uma perda de microambientes no solo levam a um colapso nos processos de decomposição, resultando em uma deficiência de carbono e outros nutrientes no solo (AYANABA et al., 1976; PHASAD et al., 1994; BASU; BEHERA, 1993).

De maneira geral, as propriedades químicas do solo avaliadas demonstraram uma melhor estruturação das áreas, conforme o aumento da idade da restauração. O mesmo foi observado por Silva et al. (2011), onde as propriedades químicas do solo encontradas em restauração com 20 anos foram semelhantes às observadas em áreas naturais, demonstrando a contribuição de restaurações na melhoria dessas propriedades do solo.

\section{CONCLUSÃO}

A comparação das áreas em processo de restauração com diferentes idades indicou uma recuperação das propriedades avaliadas ao longo do tempo. Assim, espera-se que as características de microssítio de regeneração das áreas em processo de restauração se assemelhem gradativamente às presentes nos ecossistemas de referência, sendo esse um aspecto positivo para que o recrutamento de espécies nativas seja favorecido ao longo do tempo.

\section{AGRADECIMENTOS}

À Fundação de Amparo à Pesquisa do Estado de São Paulo - FAPESP, pela bolsa concedida à primeira autora (Proc. \#2009/12663-9).

\section{REFERÊNCIAS}

AN, S. et al. Soil quality degradation processes along a deforestation chonosequence in the Ziwuling area, China. Catena, v.75, n.3, p.248-256, 2008.

ARSHAD, M. A.; LOWERY, B.; GROSSMAN, B. Physical tests for monitoring soil quality. In: DORAN, J. W.; JONES, A. J. Methods for assessing soil quality. Madison: Soil Science Society of America, 1996. p.123-141.

ARVIDSSON, J. Influence of soil texture and organic matter content on bulk density, air content, compression index and crop yield in field and laboratory experiments. Soil and Tillage Research, v.49, n.1/2, p.159-170, 1998. 
AYANABA, A.; TUCKWELL, S. B.; JENKINSON, D. S. The effects of clearing and cropping on the organic reserves and biomass of the soil. Soil Biology and

Biochemestry, v.8, n.6, p.519-525, 1976.

BARBERÁ, G. G.; NAVARRO-CANO, J. A.; CASTILLO, V. M. Seedling recruitment in a semiarid steppe: The hole of microsite and postdispersal seed predation. Journal of Arid Environments, v.67, n.4, p.701-714, 2006.

BASU, S.; BEHERA, N. The effects of tropical soil conversion on soil microbial biomass. Biology and Fertility of Soil, v.16, n.4, p.302-304, 1993.

BAVER, L. D. Practical values from physical analyses of soils. Soil Science, v.68, n.1, p.1-14, 1949.

BLAKE, G. R.; HARTGE, K. H. Bulk density. In: KLUTE, A. Methods of soil analysis physical and mineralogical methods. Madison: American Society of Agronomy/Soil Science Society of America, 1986. p.363-375.

BRADSHAW, A. D.; CHADWICK, M. J. The restoration of land: the ecology and reclamation of derelict and degraded land. Oxford: Blackwell Scientiûc Publications, 1980. 352p.

CARPENEDO, V.; MIELNICZUK, J. Estado de agregação e qualidade de agregados de Latossolos roxos, submetidos a diferentes sistemas de manejo. Revista Brasileira de Ciência do Solo, v.14, n.2, p.99-105, 1990.

DIAS JR, M. S.; PIERCE, F. J. O processo de compactação do solo e sua modelagem. Revista Brasileira de Ciência do Solo, v.20, n.2, p.175-182, 1996.

DOUST, S. J.; ERSKINE, D. P.; LAMB, D. Direct seeding to restore rainforest species: Microsite effects on the early establishment and growth of rainforest tree seedlings on degraded land in the wet tropics of Australia. Forest Ecology and Management, v.234, n.1/3, p.333-343, 2006.

ELMARSDOTTIR, A.; ARADOTTIR, A. L.; TRLICA, M. J. Microsite availability and establishment of native species on degraded and reclaimed sites. Journal of Applied Ecology, v.40, n.5, p.815-823, 2003.

\section{EMPRESA BRASILEIRA DE PESQUISA}

AGROPECUÁRIA - EMBRAPA. Centro Nacional de Pesquisa de Solos. Manual de Métodos de Análise de Solo. 2.ed. Rio de Janeiro: 1997. 212p.

FENNER, M.; THOMPSON, K. The ecology of seeds. Cambridge: Cambridge University Press, 2005. 250p.

FOWLER, N. L. Microsite requirements for germination and establishment of three grass species. American Midland Naturalist, v.115, n.1, p.131-145, 1986.

FOWLER, N. L. What is a safe site? Neighbour, litter, germination date and patch effects.

Ecology, v.69, n.4, p.947-961, 1988.

GARCIA, C.; HERNÁNDEZ, T.; COSTA, F. Microbial activity in soils under Mediterranean enviroment conditions. Soil Biology and Biochemistry, v.26, n.9, p.1185-1191, 1994.

GONÇALVES, J. L. M.; NOGUEIRA Jr., L. R.; DUCATTI, F. Recuperação de solos degradados. In: KAGEYAMA, P. Y. et al. Restauração ecológica de ecossistemas naturais. Botucatu: FEPAF, 2003. p.111-163.

GRABLE, A. R.; SIEMER, E. G. Effects of bulk density, aggregate size, and soil water suction on oxygen diffusion, redox potential and elongation of corn roots. Soil Science Society of America Journal, v.32, n.2, p.180-186, 1968.

GRUBB, P. J. The maintenance of species-richness in plant communities: the importance of the regeneration niche. Biological Reviews, v.52, n.1, p.107-145, 1977.

GUPTA, S. C.; ALLMARAS, R. R. Models to access the susceptibility of soil to excessive compaction. Advances in Soil Sciences, v.6, n.4, p.65-100, 1987.

HAJABBASI, M. A.; JALALIAN, A.; KARIMZADEH, H. R. Deforestation effects on soil physical and chemical properties, Lordegan, Iran. Plant and Soil, v.190, n.2, p.301-308, 1997.

Revista Árvore, Viçosa-MG, v.36, n.5, p.895-905, 2012 
HARPER, J. L. et al. The evolution and ecology of closely related species living in the same area. Evolution, v.15, n.2, p.209-227, 1961.

HARPER, J. L. Population biology of plants. London: Academic Press, 1977. 892p.

ISLAM, K. R.; WEIL, R. R. Land use effects on soil quality in a tropical forest ecosystem of Bangladesh. Agriculture, Ecosystems and Environment, v.79, n.1, p.9-16, 2000.

KELLMAN, M. Soil enrichment by neotropical savanna trees. Journal of Ecology, v.67, n.2, p.565-577, 1979.

KIEHL, E. J. Manual de edafologia: Relação solo-águaplanta. São Paulo: Agronômica Ceres, 1979. 262p.

KÖPPEN, W. Climatologia. Ciudad de México: Fondo Cultura Económica,. 1948.

JENNINGS, S. B.; BROWN, N. D.; SHEIL, D. Assessing forest canopies and understory illumination: canopy closure, canopy cover and other measures. Forestry, v.72, n.1, p.59-73, 1999.

LAL, R. Soil erosion problems on Alfisols in western Nigeria. Part I-V. Geoderma, v.16, n. 5, p.366-431, 1976.

LAL, R. Soil degradation and conservation of tropical rain forest. In: BOTKIN, D. B.;

CASWELL, M. F.; ESTES, J. E. Changing the global environment. Orio: Academic Press, 1989. p.135-153.

\section{LAL, R. Encyclopedia of Soil Science.}

New York: Marcel Dekker, 2002. 1476p.

LEMMON, P. E. A new instrument for measuring forest overstory density. Journal of Forestry, v.55, n.3, p.667-668, 1957.

MEIRA-NETO, J. A. A.; MARTINS, F. R.; SOUZA, A. L. Influência da cobertura e do solo na composição florística do sub-bosque em uma floresta estacional semidecidual em Viçosa, MG, Brasil. Acta Botanica Brasilica, v.19, n.3, p.473-486, 2005

MOLOFSKY, J.; AUGSPURGER, C. K. The effect of leaf litter on early seedling establishment in a tropical forest. Ecology, v.73, n.1, p.68-77, 1992.
NARAIN, P.; SINGH, R.; SINGH, K. Influence of forest cover on physio-chemical and site characteristics in Doon valley. Indian Forester, v.116, n.3, p.900-916, 1990.

NORGHAUER, J. M.; NEWBERY, D. M. Recruitment limitation after mast-seeding in two African rain forest trees. Ecology, v.91, n.8, p.2303-2312, 2010.

PARCIAK, W. Environmental variation in seed number, size, and dispersal of a fleshy-fruited plant. Ecology, v.83, n.3, p.780-793, 2002.

PÉREZ-BEJARANO, A. et al. Influence of plant species on physical, chemical and biological soil properties in a Mediterranean forest soil.

European Journal of Forest Research, v.129, n.1, p.15-24, 2010.

PHASAD, P.; BASU, S.; BEHERA, N. A comparative account of the microbiological characteristics of soils under natural forest, grassland and crop field from Eastern India. Plant and Soil, v.175, n.1, p.85-91, 1994.

REICHERT, J. M.; REINERT, D. J.; BRAIDA, J. A. Qualidade do solo e sustentabilidade de sistemas agrícolas. Ciência e Ambiente, v.27, n.1, p.29-48, 2003.

RODRIGUES, L. A. et al. Efeitos de solos e topografia sobre a distribuição de espécies arbóreas em um fragmento de floresta estacional semidecidual, em luminárias, MG. Revista Árvore, v.31, n.1, p.25-35, 2007.

RODRIGUES, R. R.; GANDOLFI, S. Conceitos, tendências e ações para a recuperação de Florestas Ciliares. In: RODRIGUES , R. R.; LEITÃO FILHO, H. F. Matas Ciliares: Conservação e Recuperação. São Paulo: Edusp, 2004. p.241-243.

RODRIGUES, R. R. et al. Large-scale ecological restoration of high-diversity tropical forests in SE Brazil. Forest Ecology and Management, v.261, n.10, p.1605-1613, 2011.

SAHANI, U.; BEHERA, N. Impact of deforestation on soil physicochemical characteristics, microbial biomass and microbial activity of tropical soil.

Land Degradation and Development, v.12, n.2, p.93-105, 2001. 
SAS Institute. The SAS system realease 8.2. Cary: 2002 .

SAXTON, K. E.; RAWLS, W. J. Soil water characteristic estimates by texture and organic matter for hydrologic solutions. Soil Science Society of America Journal, v.70, n.5, p.1569-1578, 2006.

SCARIOT, A. Seedling mortality by litterfall in amazonian forest fragments. Biotropica, v.32, n.4a, p.662-669, 2000.

SEYBOLD, C. A.; HERRICK, J. E.; BREJDA, J. J. Soil resilience: a fundamental component of soil quality. Soil Science, v.164, n.4, p.224-234, 1999.

SILVA, A. M.; MORAES, M. L. T.; BUZETTI, S. Propriedades químicas de solo sobre

florestamento ciliar após 20 anos de plantio em área de cerrado. Revista Árvore, v.35, n.1, p.97-106, 2011.

SUGANUMA, M. S. et al. Comparando metodologias para avaliar a cobertura do dossel e a luminosidade no sub-bosque de um reflorestamento e uma floresta madura. Revista Árvore, v.32, n.2, p.377-385, 2008.

SVENNING, J. C.; WRIGHT, S. J. Seed limitation in a Panamanian forest. Journal of Ecology, v.93, n.5, p.853-862, 2005.

TOPP, G. C.; ZEBCHUK, W. The determination of soil water desorption curves for soil cores.

Canadian Journal of Soil Science, v.59, n.1, p.19-26, 1979.

TURNBULL, L. A.; CRAWLEY, M. J.; REES, M. Are plant populations seed-limited? A review of seed sowing experiments. Oikos, v.88, n.2, p.225-238, 2000.
UHL, C. et al. Early plant sucession after cutting and burning in the Upper Rio Negro region of the Amazon Basin. Journal of Ecology, v.69, n.2, p.631-649, 1981.

URBANSKA, K. M. Safe sites - interface of plant population ecology and restoration ecology. In: URBANSKA, K. M.; WEBB, N. R.; EDWARDS, P. J. Restoration ecology and sustainable development. Cambridge: Cambridge University Press, 2000. p.81-110.

URIARTE, M. et al. Effects of forest fragmentation on the seedling recruitment of a tropical herb: assessing seed vs. safe-site limitation. Ecology, v.91, n.5, p.1317-1328, 2010.

VELOSO, H. P. Manual técnico da vegetação brasileira. Rio de Janeiro: IBGE, 1992.

VOMOCIL, J. A.; FLOCKER, W. J. Effect of soil compaction on storage and movement of soil air and water. Transactions of the American Society of Agricultural Engineers, v.4, n.1, p.242-246, 1961.

ZHANG, F. et al. The impacts of accelerated erosion on soil nutrient loss following deforestation in the Ziwuling area. Plant Nutrition and Fertilizer Science, v.12, n.6, p.826-821, 2006.

ZHENG, F. L. Effects of accelerated soil erosion on soil nutrient loss after deforestation on the loess plateau. Pedosphere, v.15, n.6, p.707-715, 2005.

ZHENG, F. L. et al. Relationship of ecoenvironmental change with natural erosion and artificially accelerated erosion. The Journal of China Geography, v.7, n.2, p.75-84, 1997. 
